\title{
Robust Actor-Critic Contextual Bandit for Mobile Health (mHealth) Interventions
}

\author{
Feiyun Zhu \\ Department of CSE, \\ University of Texas at Arlington, TX \\ fyzhu0915@gmail.com \\ Ruoyu Li \\ Department of CSE, \\ University of Texas at Arlington, TX \\ ruoyu.li@mavs.uta.edu
}

\author{
Jun Guo \\ Department of Statistics, \\ University of Michigan, Ann Arbor, MI \\ guojun@umich.edu \\ Junzhou Huang* \\ University of Texas at Arlington, \\ Tencent AI Lab \\ jzhuang@uta.edu
}

\begin{abstract}
We consider the actor-critic contextual bandit for the mobile health (mHealth) intervention. State-of-the-art decision-making algorithms generally ignore the outliers in the data-set. In this paper, we propose a novel robust contextual bandit method for the mHealth. It can achieve the conflicting goal of reducing the influence of outliers, while seeking for a similar solution compared with the state-ofthe-art contextual bandit methods on the datasets without outliers. Such performance relies on two technologies: (1) the capped- $\ell_{2}$ norm; (2) a reliable method to set the threshold hyper-parameter, which is inspired by one of the most fundamental techniques in the statistics. Although the model is non-convex and non-differentiable, we propose an effective reweighted algorithm and provide solid theoretical analyses. We prove that the proposed algorithm can sufficiently decrease the objective function value at each iteration and will converge after a finite number of iterations. Extensive experiment results on two datasets demonstrate that our method can achieve almost identical results compared with state-of-the-art contextual bandit methods on the dataset without outliers, and significantly outperform those state-of-the-art methods on the badly noised dataset with outliers in a variety of parameter settings.
\end{abstract}

\section{CCS CONCEPTS}

- Theory of computation $\rightarrow$ Reinforcement learning; Sequential decision making; Markov decision processes; • Computing methodologies $\rightarrow$ Machine learning algorithms; • Applied computing $\rightarrow$ Health care information systems;

*This work was partially supported by NSF IIS-1423056, CMMI-1434401, CNS-1405985, IIS-1718853 and the NSF CAREER grant IIS-1553687.

Permission to make digital or hard copies of all or part of this work for personal or classroom use is granted without fee provided that copies are not made or distributed for profit or commercial advantage and that copies bear this notice and the full citation on the first page. Copyrights for components of this work owned by others than ACM must be honored. Abstracting with credit is permitted. To copy otherwise, or republish, to post on servers or to redistribute to lists, requires prior specific permission and/or a fee. Request permissions from permissions@acm.org.

ACM-BCB'18, August 29-September 1, 2018, Washington, DC, USA

(C) 2018 Association for Computing Machinery.

ACM ISBN 978-1-4503-5794-4/18/08 .. \$15.00

https://doi.org/10.1145/3233547.3233553

\section{KEYWORDS}

Robust Learning, Actor-Critic Contextual Bandit, Markov Decision Process (MDP), Mobile Health (mHealth) Intervention.

ACM Reference format:

Feiyun Zhu, Jun Guo, Ruoyu Li, and Junzhou Huang. 2018. Robust ActorCritic Contextual Bandit for Mobile Health (mHealth) Interventions. In Proceedings of 9th ACM International Conference on Bioinformatics, Computational Biology and Health Informatics, Washington, DC, USA, August 29-September 1, 2018 (ACM-BCB'18), 10 pages.

https://doi.org/10.1145/3233547.3233553

\section{INTRODUCTION}

Due to the explosive growth of smart device (i.e. smartphones and wearable devices, such as Fitbit etc.) users globally, mobile health (mHealth) technologies draw increasing interests from the scientific community $[22,24]$. The goal of mHealth is to deliver in-time interventions to device users, guiding them to lead healthier lives, such as reducing alcohol abuse $[2,8,11,14,30,33]$ and increasing physical activities $[1,20]$. With the advanced smart technologies, the mHealth interventions can be formed according to the users' ongoing statuses and changing needs, which is more portable and flexible compared with the traditional treatments. Therefore, mHealth technologies are widely used in lots of health-related applications, such as eating disorders, alcohol abuses, mental illness, obesity management and HIV medication adherence [19, 22, 24].

Formally, the tailoring of mHealth intervention is modeled as a sequential decision-making (SDM) problem. The contextual bandit algorithm provides a framework for the SDM [30, 41, 42] . In 2014, Lei [17] proposed the first contextual bandit algorithm for the mHealth study. It is in the actor-critic setting [29], which has two advantages compared with the critic only contextual bandit methods for the internet advertising [18]: (a) Lei's method has an explicit parameterized model for the stochastic policy. By analyzing the estimated parameters in the learned policy, we could know the key features that contribute most to the policy. This is important to the behavior scientists for the state (feature) design. (b) From the perspective of optimization, the actor-critic algorithm has great properties of quick convergence with low variance [10].

However, Lei's method assumes that the states at different decision points are i.i.d. and the current action only influences the 
immediate reward [16]. This assumption is infeasible in real situations. Taking the delayed effect in the SDM or mHealth for example, the current action influences not only the immediate reward but also the next state and through that, all the subsequent rewards [29]. Accordingly, Lei proposed a new method [16] by emphasizing on explorations and reducing exploitations.

Although those two methods serve a good start for the mHealth study, they assume that the noise in the trajectory follows the Gaussian distribution. The least square based algorithm is employed to estimate the expected reward. In reality, however, there are various kinds of complex noises that can badly degrade the collected data, for example: (1) the wearable device is unreliable to accurately record the states and rewards from users under different conditions. (2) The mobile network is unavailable in some areas. Such case hinders the collecting of users' states as well as the sending of interventions. (3) The mHealth relies on the self-reported information (Ecological Momentary Assessments, i.e. EMAs) [8] to deliver effective interventions. However, some users are annoyed at the EMAs. They either fill out the EMAs via random selections or just leave some or all the EMAs blank. We consider the various kinds of badly noised observations in the trajectory as outliers.

There are several robust methods for the SDM problem [7, 34, 39]. However, those methods are neither in the actor-critic setting, nor focusing on the outlier problem. Thus, they are different from this paper's focus. In the general machine learning task, there are some robust learning methods to deal with the outlier problem $[6,13$, $27,31,35,37,43]$. However, none of them are contextual bandit algorithms-it may cost of lots of work to transfer their methods to the (actor-critic) contextual bandit algorithms. Besides, those methods seldom pay attention to the dataset without outliers. In practice, however, we don't know whether a given dataset consists of outliers or not. It is necessary to propose a robust learning method that works well on the dataset both with and without outliers.

To alleviate the above problems, we propose a robust contextual bandit method for the mHealth. The capped- $\ell_{2}$ norm is used to measure the learning error for the expected reward estimation (i.e. the critic updating). It prevents outlier observations from dominating our objective. Besides, the learned weights in the critic updating are considered in the actor updating. As a result, the robustness against outliers are greatly boosted in both actor-critic updatings. There is an important thresholding parameter $\epsilon$ in our method. We propose a solid method to set its value according to the distribution of samples, which is based on one of the most fundamental ideas in the statistics. It has two benefits: (1) the setting of $\epsilon$ becomes very easy and reliable; (2) we may achieve the conflicting goal of reducing the influence of outliers when the dataset indeed contains outliers, while achieving almost identical results compared with the state-of-the-art contextual bandit method on the datasets without outliers. Although the objective is non-convex and nondifferentiable, we derivate an effective algorithm. As a theoretical contribution, we prove that our algorithm could find sufficiently decreasing point after each iteration and finally converges after a finite number of iterations. Extensive experiment results on two datasets verify that our methods could achieve clear gains over the state-of-the-art contextual bandit methods for the mHealth.

\section{PRELIMINARIES}

Multi-armed bandit (MAB) is the simplest algorithm for the sequential decision making problem (SDM). The contextual bandit is a more practical extension of the MAB by considering some extra information that is helpful for the SDM problem [30]. The use of context information allows for many interesting applications, such as internet advertising and health-care tasks [7, 30, 36, 38, 41].

In contextual bandit, the expected reward is an core concept that measures how many rewards we may on average get when it is in state $s$ and choosing action $a$, i.e., $\mathbb{E}(r \mid s, a)$. Since the state space is usually very large or even infinite in the mHealth tasks, the parameterized model is employed to approximate the expected reward:

$$
\mathbb{E}(r \mid s, a)=\mathbf{x}(s, a)^{\top} \mathbf{w},
$$

where $\mathbf{x}(s, a) \in \mathbb{R}^{u}$ is a feature processing step that combines information in the state $s$ and the action $a, \mathbf{w} \in \mathbb{R}^{u}$ is the unknown coefficient vector.

In 2014, Lei [17] proposed the first contextual bandit method for the mHealth study. It is in the actor-critic learning setting. The actor updating is the overall optimization goal. It aims to learn an optimal policy $\pi^{*}$ that maximizes the average rewards over all the states and actions [10]. The objective function is

$$
\pi_{\theta_{n}^{*}}=\arg \max _{\theta_{n}} \widehat{J}\left(\theta_{n}\right)
$$

for the $n^{\text {th }}$ user, where

$$
\widehat{J}\left(\theta_{n}\right)=\sum_{s \in \mathcal{S}} d_{\mathrm{ref}}^{(n)}(s) \sum_{a \in \mathcal{A}} \pi_{\theta_{n}}(a \mid s) \mathbb{E}(r \mid s, a)
$$

and $d_{\text {ref }}^{(n)}(s)$ is a reference distribution over states for user $n$.

Obviously, we need the estimation of expected rewards $\mathbb{E}(r \mid s, a)$ to define the objective (1) for the actor updating. Such procedure is called the critic updating (i.e. policy evaluation). State-of-theart method generally employs the ridge regression to learn the expected reward from the observations. The objective is

$$
\min _{\mathbf{w}_{n}} \sum_{\mathcal{U}_{i} \in \mathcal{D}_{n}}\left\|\mathbf{x}\left(s_{i}, a_{i}\right)^{\top} \mathbf{w}_{n}-r_{i}\right\|_{2}^{2}+\zeta_{c}\left\|\mathbf{w}_{n}\right\|_{2}^{2},
$$

where

$$
\mathcal{D}_{n}=\left\{\mathcal{U}_{i}=\left(s_{i}, a_{i}, r_{i}\right) \mid i=0, \cdots, M\right\}
$$

is the trajectory of observed tuples from the $n^{\text {th }}$ user and $\mathcal{U}_{i}$ is the ${ }^{\text {th }}$ tuple in $\mathcal{D}_{n} ; \zeta_{c}$ is a tuning parameter to control the strength of constraints. It has a closed-form solution for (2) as:

$$
\widehat{\mathbf{w}}_{n}=\left(\mathbf{X}_{n} \mathbf{X}_{n}^{\top}+\zeta_{c} \mathbf{I}_{u}\right)^{-1} \mathbf{X}_{n} \mathbf{r}_{n} .
$$

where $\mathbf{I}_{u}$ is a $u \times u$ identity matrix. Unfortunately, similar to the existing least square based models in machine learning and statistics, the objective function in (2) is prone to the presence of outliers $[25,40]$.

\section{ROBUST ACTOR-CRITIC CONTEXTUAL BANDIT VIA THE CAPPED- $\ell_{2}$ NORM}

To enhance the robustness in the critic updating, the capped- $\ell_{2}$ norm based measure is used for the estimation of expected rewards. By imposing the learned weights for the actor updating, we propose a robust objective for the actor updating. 


\subsection{Robust Critic Updating via the Capped- $\ell_{2}$ Norm}

To simplify the notation, we get rid of the subscript index $n$, which is used to indicate the model for the $n^{\text {th }}$ user. The new objective for the critic updating (i.e. policy evaluation) is

$$
\min _{\mathbf{w}} \sum_{i=1}^{M} \min \left\{\left\|r_{i}-\mathbf{x}_{i}^{T} \mathbf{w}\right\|_{2}^{2}, \epsilon\right\}+\zeta_{c}\|\mathbf{w}\|_{2}^{2},
$$

where $\min \left\{\|y\|_{2}^{2}, \epsilon\right\}$ is the capped- $\ell_{2}$ norm for a vector $\mathbf{y} ; \epsilon>0$ is the threshold hyper-parameter to choose the effective observations for the critic updating; $\mathbf{x}_{i}=\mathbf{x}\left(s_{i}, a_{i}\right)$ is the feature for the estimation of expected rewards.

If the residual of the $i^{\text {th }}$ tuple is

$$
\left\|r_{i}-\mathbf{x}_{i}^{\top} \mathbf{w}\right\|_{2}^{2}>\epsilon
$$

we treat it as an outlier. Its residual is capped to a fixed value $\epsilon$. That is, the influence of the $i^{\text {th }}$ tuple is fixed [9], which cannot cause bad influences on the learning procedure. For the tuples whose residuals satisfy

$$
\left\|r_{i}-\mathbf{x}_{i}^{\top} \mathbf{w}\right\|_{2}^{2} \leq \epsilon
$$

we consider them as effective observations and keep them as they are in the optimization process.

Therefore, it is extremely important to properly set the value of $\epsilon$. When $\epsilon$ is too large, the outliers that distribute far away from the majority of tuples will be treated as effective samples, causing bad influences to the learning procedure. When $\epsilon$ is too small, most tuples are treated as outliers-there would be very few of effective samples for the cirtic learning. Such case easily leads to some unstable policies that contain lots of variances. Specially if $\epsilon \rightarrow+\infty$, our objective is equivalent to the least square objective in (2).

One of our major contribution is that we propose a reliable method to properly set the value of $\epsilon$. Our method does not need any specific assumption on the data distribution. It is derivated from the boxplot-one of the most fundamental ideas in the statistics $[4,32]$. To give a descriptive illustration of the data distribution, the boxplot is widely used by specifying 5 points, including the min, lower quartile $q_{1}$, median, upper quartile $q_{3}$ and max. Based on the 5 points, the boxplot provides a method to detect outliers. Following this idea, we set the value of $\epsilon$ as

$$
\epsilon=\tau\left(q_{3}+1.5 \times I Q R\right)
$$

where

$$
I Q R=q_{3}-q_{1}
$$

is the interquartile range; $\tau$ is introduced only for the experiment setting S2, otherwise we may ignore the parameter $\tau$ by keeping it fixed at 1 . Intuitively, the data points that are $1.5 \times I Q R$ more above the third quartile are detected as the outliers. Compared with the state-of-the-art robust learning methods $[9,13,27]$ in the other fields that have to manually set the thresholding hyper-parameter, we provide an adaptive method to set $\epsilon$, which is well adapted to the data distribution.

With the capped- $\ell_{2}$ norm and the method to set $\epsilon$ in (5), we can achieve the conflicting goals of (a) reducing the influence of outliers when the dataset has outliers, while (b) seeking for a similar solution compared with that of the state-of-the-art method if there is no outlier in the dataset. As a result, our method can deal with various datasets, regardless of whether they consist of outliers or not.

\subsection{Derivation of a General Objective Function for the Objective (4)}

To derive an efficient algorithm, we consider a more general capped$\ell_{2}$ norm based objective for (4) as follows

$$
\min _{\mathbf{x}} \sum_{i=1}^{M} \min \left\{\left\|h_{i}(\mathbf{x})\right\|_{2}^{2}, \epsilon\right\}+\zeta g(\mathbf{x}),
$$

where $\|\cdot\|_{2}^{2}$ is the $\ell_{2}$ norm for a vector; $h_{i}(\mathbf{x})$ and $g(\mathbf{x})$ are both scalar functions of $\mathbf{x}$. In this section, we propose an iteratively re-weighted method to simplify the objective (6).

Due to the non-smooth and non-differentiable property of (6), we could only obtain the sub-gradient of (6) as:

$$
\partial O(\mathbf{x})=\sum_{i=1}^{M} \partial \min \left(\left\|h_{i}(\mathbf{x})\right\|_{2}^{2}, \epsilon\right)+\zeta \partial g(\mathbf{x}),
$$

where $O(\mathbf{x})$ is the representation of the objective function (4). Letting

$$
f=\partial \min \left(\left\|h_{i}(\mathbf{x})\right\|_{2}^{2}, \epsilon\right)
$$

gives

$$
f= \begin{cases}0 & \text { if }\left\|h_{i}(\mathbf{x})\right\|_{2}^{2}>\epsilon \\ 2\left[d_{\text {low }}, d_{\text {high }}\right] \partial h_{i}(\mathbf{x}) & \text { if }\left\|h_{i}(\mathbf{x})\right\|_{2}^{2}=\epsilon, \\ 2 h_{i}(\mathbf{x}) \partial h_{i}(\mathbf{x}) & \text { if }\left\|h_{i}(\mathbf{x})\right\|_{2}^{2}<\epsilon\end{cases}
$$

where $d_{\text {low }}=\min \left\{0, h_{i}(\mathbf{x})\right\}$ and $d_{\text {high }}=\max \left\{0, h_{i}(\mathbf{x})\right\}$. For the sake of easy optimization, we provide a compact expression that satisfies the sub-gradient in (8) by introducing a variable $u_{i}=$ $1_{\left\{\left\|h_{i}(\mathrm{x})\right\|_{2}^{2}<\epsilon\right\}}$. Then Eq. (7) is rewritten as

$$
\partial O(\mathbf{x})=2 \sum_{i} u_{i} h_{i}(\mathbf{x}) \partial h_{i}(\mathbf{x})+\zeta \partial g(\mathbf{x}) .
$$

Since $u_{i}$ depends on $\mathbf{x}$, it is very challenging to directly solve the objective (9). Once $u_{i}$ is given for every $i$, the objective (7) is equivalent to the following problem

$$
\min _{\mathbf{x}} \sum_{i=1}^{M} u_{i}\left\|h_{i}(\mathbf{x})\right\|_{2}^{2}+\zeta g(\mathbf{x})
$$

in the sense that they have the same partial derivative.

\subsection{Robust Algorithm for the Critic Updating}

In this section, we provide an effective updating rule for the objective function (4) (cf. Proposition 1 and Algorithm 1). We prove that our algorithm can find sufficiently decreasing point after each iteration (cf. Lemma 3.1) and finally converge after a finite number of iterations (cf. Theorem 1).

Proposition 1. The iterative updating rule $(4)(\forall t>1)$ is

$$
\mathbf{w}^{(t)}=\left(\mathrm{XU}^{(t-1)} \mathbf{X}^{\top}+\zeta_{c} \mathbf{I}\right)^{-1} \mathrm{XU}^{(t-1)} \mathbf{r}
$$

where

$$
\mathbf{U}^{(t)}=\operatorname{diag}\left(\mathbf{u}^{(t)}\right) \in \mathbb{R}^{M \times M}
$$


is a nonnegative diagonal matrix. The $i^{\text {th }}$ element is

$$
u_{i}^{(t)}=1_{\left\{\left\|r_{i}-\mathbf{x}_{i}^{\top} \widehat{\mathbf{w}}^{(t)}\right\|_{2}^{2}<\epsilon\right\}} \cdot
$$

Lemma 3.1. The updating rule in Proposition 1 leads to sufficient decrease of the objective function $O(\mathbf{w})$ in (4):

$$
F\left(\mathbf{w}^{(t-1)}, \mathbf{u}^{(t-1)}\right) \geq F\left(\mathbf{w}^{(t)}, \mathbf{u}^{(t)}\right)+\zeta\left\|\mathbf{w}^{(t)}-\mathbf{w}^{(t-1)}\right\|_{2}^{2},
$$

We have used the bivariate function $F(\mathbf{w}, \mathbf{u})$ to substitute $O(\mathbf{w})$ in notation, which is defined as

$$
F(\mathbf{w}, \mathbf{u})=\sum_{i}^{M} u_{i}\left\|r_{i}-\mathbf{x}_{i}^{T} \mathbf{w}\right\|_{2}^{2}+\sum_{i}^{M}\left(1-u_{i}\right) \epsilon+\zeta_{c}\|\mathbf{w}\|_{2}^{2},
$$

Notice that $F(\mathbf{w}, \mathbf{u})$ denotes the same function as $O(\mathbf{w})$ in (4). Here we introduce the bivariate notation since

$$
\mathbf{u}=1_{\left\{\left\|r_{i}-\mathbf{x}_{i}^{T} \mathbf{w}\right\|_{2}^{2}<\epsilon\right\}}
$$

involves the unknown parameter $\mathbf{w}$ and will be simultaneously updated with $\mathbf{w}$ in the algorithm.

Lemma 3.2. For $\left\{\mathbf{w}^{(t)}, t \geq 0\right\}$ in Lemma 3.1, we show that

$$
\sum_{t=1}^{\infty}\left\|\mathbf{w}^{(t)}-\mathbf{w}^{(t-1)}\right\|_{2}^{2}<\infty
$$

and consequently

$$
\lim _{t \rightarrow \infty}\left\|\mathbf{w}^{(t)}-\mathbf{w}^{(t-1)}\right\|_{2}^{2}=0 .
$$

Theorem 1. The updating rule in Proposition 1 converges to a local optimum of the objective function $O(w)$ in 4 .

The proofs for Proposition 1, Lemma 3.1, Lemma 3.2 and Theorem 1 are given in the appendixes, which are in the end of the paper.

\subsection{Robust Actor Updating for the Stochastic Policy}

Besides the critic updating, outliers can also badly influence the actor updating in (1), which is our ultimate objective for the actorcritic contextual bandit. To boost its robustness, the estimated weights learned in the critic updating are considered. Since $d_{\text {ref }}(s)$ is usually unavailable in reality, the $M$-trial based objective [3] is widely used. Thus, the objective (1) is rewritten as

$$
\widehat{J}(\theta)=\frac{u_{i}}{M} \sum_{i=1}^{M} \sum_{a \in \mathcal{A}} \pi_{\theta}\left(a \mid s_{i}\right) \mathbb{E}\left(r \mid s_{i}, a\right)-\frac{\zeta_{a}}{2}\|\theta\|_{2}^{2},
$$

where

$$
\mathbb{E}\left(r \mid s_{i}, a\right)=\mathbf{x}\left(s_{i}, a\right)^{\top} \widehat{\mathbf{w}}
$$

is the estimated expected reward; $\|\theta\|_{2}^{2}$ is the least square constraint to make the objective (12) a well-posed problem and $\zeta_{a}$ is a balancing parameter that controls the penalization strength [17].

Compared with the current objective for the actor updating in (1), our objective has an extra weight term $\left\{u_{i}\right\}_{i=1}^{M}$, which gives those tuples, whose residuals are very large in the critic updating, zero weights. As a result, the outlier tuples that are far away from

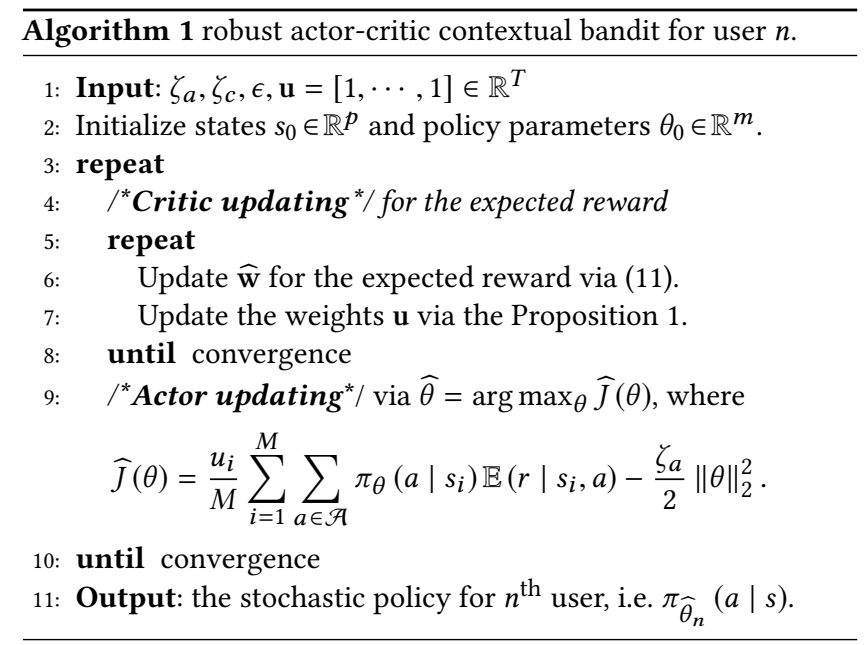

the majority of tuples are removed from the actor updating, enhancing the robustness. The algorithm of the actor updating performs the maximization of (12) over $\theta$. This is learned via the Sequential Quadratic Programming (SQP) algorithm. We utilize the implementation of SQP with finite-difference approximation to the gradient in the fmincon function of Matlab. The SQP consists of three main stages: (1) updating the hessian matrix, (2) quadratic programming solution, and (3) line search and merit function [26]. Besides, the $\mathrm{SQP}$ is also used to optimize the objective.

\section{EXPERIMENT}

\subsection{Two Datasets}

Two datasets are used to verify the performance of our method. The first dataset is on the personalizing treatment delivery in mobile health, which is a common application of the sequential decision making algorithm [24]. In this paper, we focus on the Heartsteps, where the participants are periodically sent activity suggestions aimed at decreasing sedentary behavior [14]. Specifically, Heartsteps is a 42-days trial study where 50 participants are involved. For each participant, there are 210 decision points-five decisions per participant per day. At each time point, the set of intervention actions can be the intervention type, as well as whether or not to send interventions. The intervention actions generally depend on the state of the participant as well as the formerly sent interventions. Interventions can be sent via smartphones, or via other wearable devices like a wristband [5].

The Heartsteps is a common application for the contextual bandit algorithm [16, 24]. The goal is to learn an optimal SDM algorithms to decide what type of intervention actions to send to each user to maximize the cumulative steps each user takes. The resulting data for each participant is a sequence

$$
\mathcal{D}=\left\{s_{0}, a_{0}, r_{0}, \cdots, s_{209}, a_{209}, r_{209}, r_{210}, s_{210}\right\},
$$

where $s_{t}$ is the participant's state at time $t$. It is a three dimensional vector that consists of (1) the weather condition, (2) the engagement of participants, (3) the treatment fatigue of participants. $a_{t}$ indicates whether or not to send the intervention to users; since the goal of Heartsteps is to increase the participant's activities, we define the 
Table 1: The ElrAR of nine contextual methods on the two datasets: Heartsteps and chain walk. (experiment setting S1)

\begin{tabular}{|c|ccc|}
\cline { 2 - 4 } \multicolumn{1}{c|}{} & \multicolumn{3}{c|}{ Long-run Average Rewards of 9 methods on 2 datasets } \\
\hline Datasets & LinUCB & OutlierFilter + LinUCB & Ro-LinUCB \\
\hline HeartSteps & $1391.25 \pm 49.45$ & $1447.60 \pm 41.98$ & $\mathbf{1 5 7 8 . 9 7} \pm \mathbf{1 3 . 7 2}$ \\
Chian walk & $35.910 \pm 7.744$ & $41.903 \pm 5.840$ & $\mathbf{4 3 . 9 4 2} \pm \mathbf{7 . 2 9 0}$ \\
\hline \hline Datasets & ACCB & OutlierFilter + ACCB & Ro-ACCB \\
\hline HeartSteps & $1383.19 \pm 50.40$ & $1442.41 \pm 42.30$ & $\mathbf{1 5 7 8 . 6 3} \pm \mathbf{1 2 . 6 5}$ \\
Chian walk & $41.054 \pm 5.517$ & $44.521 \pm 7.878$ & $\mathbf{4 9 . 5 1 7} \pm \mathbf{4 . 2 2 4}$ \\
\hline \hline Datasets & SACCB & OutlierFilter + SACCB & Ro-SACCB \\
\hline HeartSteps & $1386.57 \pm 49.01$ & $1427.46 \pm 45.32$ & $\mathbf{1 5 6 7 . 3 3} \pm \mathbf{1 2 . 9 6}$ \\
Chian walk & $40.739 \pm 5.628$ & $42.123 \pm 6.569$ & $\mathbf{4 7 . 0 7 8} \pm \mathbf{4 . 8 3 4}$ \\
\hline
\end{tabular}

reward, $r_{t}$, as the step count for the 3 hours following a decision point.

The second dataset is the 4-state chain walk, which is a benchmark dataset for the (contextual) bandit and reinforcement learning study. Please refer to [15] for the details of the chain walk dataset. The reward vector over the four states is $(0,100,100,0)$ in this paper.

\subsection{Nine Compared Methods}

There are nine contextual bandit methods compared in the experiment, including three state-of-the-art methods: (1) Linear Upper Confidence Bound Algorithm (LinUCB) is one of the most famous contextual bandit methods used in the personalized news recommendation $[12,18]$; (2) the actor-critic contextual bandit (ACCB) is the first SDM method for the mHealth intervention [17]; (3) the stochasticity constrained actor-critic contextual bandit (SACCB) for the mHealth [16]. We improve the above three methods by first using the state-of-the-art outlier filter $[11,23]$ to remove outliers, then employing the above three contextual bandit algorithms for the SDM task. In the $7^{\text {th }}$ to $9^{\text {th }}$ compared methods, we apply the proposed robust model and optimization algorithms in the three state-of-the-art contextual bandit methods, leading to (7) Robust LinUCB (Ro-LinUCB for short), (8) Robust ACCB (Ro-ACCB), (9) Robust SACCB (Ro-SACCB).

\subsection{Evaluation Methodology and Parameter Setting}

It has been a challenging problem to reliably evaluate the sequential decision making (e.g., bandit and reinforcement learning) algorithms when the simulator (like the Atari games) is unavailable $[18,21]$. After the model is trained, we use it to interact with the enviroment (or simulator) to collect thousands of immediate rewards for the calculation of the long term rewards as the evaluation metric. However for a wide variety of applications including our HeartSteps, the simulator is unavailable. In this paper, we use a benchmark evalutaion methods [21]. The main idea is to make use of the collected dataset to build a simulator, based on which we train and evaluate the contextual bandit methods. Such case makes it impossible to train and evaluate the contextual bandit algorithms on up to ten datasets like the general supervised learning tasks.

In the Heartsteps study, there are 50 users; the simulator for each user is as follows: the initial state is drawn from the Gaussian distribution $S_{0} \sim \mathcal{N}_{p}\{0, \Sigma\}$, where $\Sigma$ is a $p \times p$ covariance matrix with pre-defined elements and $p$ (3, say) is the dimension of the state. For $t \geq 0, a_{t}$ is drawn from the learned policy $\pi_{\widehat{\theta}_{n}}\left(a_{t} \mid s_{t}\right)$ during the evaluation procedure. When $t \geq 1$, the state and immediate reward are generated as

$$
\begin{aligned}
S_{t, 1}= & \beta_{1} S_{t-1,1}+\xi_{t, 1}, \\
S_{t, 2}= & \beta_{2} S_{t-1,2}+\beta_{3} A_{t-1}+\xi_{t, 2}, \\
S_{t, 3}= & \beta_{4} S_{t-1,3}+\beta_{5} S_{t-1,3} A_{t-1}+\beta_{6} A_{t-1}+\xi_{t, 3}, \\
R_{t}= & \beta_{13} \times\left[\beta_{7}+A_{t} \times\left(\beta_{8}+\beta_{9} S_{t, 1}+\beta_{10} S_{t, 2}\right)\right. \\
& \left.+\beta_{11} S_{t, 1}-\beta_{12} S_{t, 3}+\varrho_{t}\right],
\end{aligned}
$$

where $\boldsymbol{\beta}=\left\{\beta_{1}, \cdots, \beta_{13}\right\}=[0.4,0.3,0.4,0.7,0.05,0.6,3,0.25,0.25,0.4,0.1$, $0.5,500]$ is the main parameter for the MDP system.

$$
\left\{\xi_{t, i}\right\}_{i=1}^{p} \sim \mathcal{N}(0,1),
$$

$p=3$ is the number of states and $\varrho_{t} \sim \mathcal{N}(0,9)$ are the Gaussian noises in the state (13) and in the reward (14) respectively. There are two steps to simulate the outliers in the trajectory: (a) randomly select a fixed ratio ( $\psi=4 \%$, say) of tuples in each user's trajectory; (b) a large noise ( $v$ times the average value in the trajectory) is added to the states and rewards in the selected tuples. Besides, the actions in the selected tuples are randomly set to simulate the random failure of sending interventions due to the weak mobile network.

The parameterized policy is assumed to follow the Boltzmann distribution

$$
\pi_{\theta}(a \mid s)=\frac{\exp \left[-\theta^{\top} \phi(s, a)\right]}{\sum_{a^{\prime}} \exp \left[-\theta^{\top} \phi\left(s, a^{\prime}\right)\right]},
$$

where

$$
\phi(s, a)=\left[a s^{\top}, a\right]^{\top} \in \mathbb{R}^{m}
$$

(where $m=p+1$ ) is the policy feature, $\theta \in \mathbb{R}^{4}$ is the unknown coefficient vector. The feature for the estimation of expected rewards is set

$$
\mathbf{x}(s, a)=\left[1, s^{\top}, a, s^{\top} a\right]^{\top} \in \mathbb{R}^{u},
$$

where $u=2 p+2$. The $\ell_{2}$ constraint for the actor-critic learning is set as $\zeta_{a}=\zeta_{c}=0.001$. The outlier ratio and strength are set $\psi=4 \%$ and $v=5$ respectively. In our methods, $\tau$ is set as 1 by default.

The expected long-run average reward (ElrAR) [24] is used to quantify the quality of the estimated policy $\pi_{\widehat{\theta}_{n}}$ for $n \in\{1, \cdots, 50\}$. Intuitively, ElrAR measures the average steps users take per day in 
Table 2: The ElrAR of six methods vs. outlier ratio $\psi$ on two datasets: (1) Heartsteps and (2) chain walk. (experiment S2).

\begin{tabular}{|c|c|c|c|c|c|c|}
\hline \multirow{2}{*}{$\begin{array}{c}\text { outlier } \\
\text { ratio }\end{array}$} & \multirow[b]{2}{*}{ LinUCB } & \multicolumn{4}{|c|}{ Expected Long-run Average Rewards (ElrAR) on the HeartSteps dataset } & \multirow[b]{2}{*}{ Ro-SACCB } \\
\hline & & Ro-LinUCB & $\mathrm{ACCB}$ & Ro-ACCB & SACCB & \\
\hline $0 \%$ & $1578.98 \pm 13.73$ & $1579.03 \pm 13.73$ & $1578.31 \pm 12.70$ & $1578.31 \pm 12.55$ & $1574.18 \pm 12.56$ & $1550.27 \pm 20.66$ \\
\hline $1 \%$ & $1462.68 \pm 40.20$ & $1578.97 \pm 13.74$ & $1462.93 \pm 39.87$ & $1578.38 \pm 12.61$ & $1458.19 \pm 40.64$ & $1552.48 \pm 20.57$ \\
\hline $3 \%$ & $1428.25 \pm 49.67$ & $1578.96 \pm 13.73$ & $1429.55 \pm 45.80$ & $1578.21 \pm 12.57$ & $1429.12 \pm 45.56$ & $1555.94 \pm 20.14$ \\
\hline $5 \%$ & $1391.25 \pm 49.45$ & $1578.97 \pm 13.72$ & $1383.19 \pm 50.40$ & $1578.63 \pm 12.66$ & $1386.57 \pm 49.01$ & $1567.33 \pm 12.96$ \\
\hline $7 \%$ & $1370.52 \pm 50.23$ & $1578.96 \pm 13.73$ & $1365.85 \pm 49.09$ & $1578.68 \pm 12.62$ & $1368.26 \pm 48.28$ & $1569.31 \pm 12.57$ \\
\hline $9 \%$ & $1359.07 \pm 48.40$ & $1579.05 \pm 13.72$ & $1351.63 \pm 46.19$ & $1578.66 \pm 12.72$ & $1355.21 \pm 45.28$ & $1570.72 \pm 12.72$ \\
\hline mean & 1431.79 & 1578.99 & 1428.44 & 1578.48 & 1435.37 & 1561.01 \\
\hline outlier & \multicolumn{5}{|c|}{ Expected Long-run Average Rewards (ElrAR) on the chain walk dataset } & \multirow[b]{2}{*}{ Ro-SACCB } \\
\hline ratio & LinUCB & Ro-LinUCB & $\mathrm{ACCB}$ & Ro-ACCB & SACCB & \\
\hline $0 \%$ & $\mathbf{4 7 . 0 6 1} \pm 6.714$ & $46.904 \pm 6.932$ & $52.191 \pm 4.545$ & $\mathbf{5 2 . 2 0 8} \pm \mathbf{4 . 5 5 4}$ & $52.215 \pm 4.669$ & $52.222 \pm 4.663$ \\
\hline $1 \%$ & $27.646 \pm 6.186$ & $46.752 \pm 7.020$ & $31.368 \pm 5.123$ & $50.776 \pm 4.819$ & $30.407 \pm 5.143$ & $49.670 \pm 5.103$ \\
\hline $3 \%$ & $33.930 \pm 6.485$ & $47.038 \pm 7.485$ & $35.213 \pm 5.138$ & $51.108 \pm 4.670$ & $34.005 \pm 5.188$ & $49.486 \pm 5.093$ \\
\hline $5 \%$ & $35.910 \pm 7.744$ & $43.942 \pm 7.290$ & $41.054 \pm 5.517$ & $49.517 \pm 4.224$ & $40.739 \pm 5.628$ & $\mathbf{4 7 . 0 7 8} \pm \mathbf{4 . 8 3 4}$ \\
\hline $7 \%$ & $31.490 \pm 6.956$ & $40.955 \pm 7.857$ & $38.856 \pm 4.385$ & $46.295 \pm 4.671$ & $38.393 \pm 4.481$ & $45.040 \pm 5.035$ \\
\hline $9 \%$ & $29.952 \pm 6.905$ & $\mathbf{3 7 . 9 5 8} \pm 6.935$ & $38.030 \pm 4.200$ & $41.637 \pm 6.032$ & $37.724 \pm 4.383$ & $40.939 \pm 6.100$ \\
\hline mean & 34.331 & 43.925 & 39.452 & 48.590 & 38.914 & 47.406 \\
\hline
\end{tabular}

Table 3: The ElrAR of six methods vs. outlier strength $v$ on two datasets: (1) Heartsteps and (2) chain walk. (experiment S3).

\begin{tabular}{|c|c|c|c|c|c|c|}
\hline \multirow{2}{*}{$\begin{array}{c}\text { outlier } \\
\text { strength }\end{array}$} & \multicolumn{6}{|c|}{ Expected Long-run Average Rewards (ElrAR) on the HeartSteps dataset } \\
\hline & LinUCB & Ro-LinUCB & ACCB & Ro-ACCB & SACCB & Ro-SACCB \\
\hline $0 \times$ & $1578.98 \pm 13.75$ & $1579.03 \pm 13.77$ & $1578.31 \pm 12.70$ & $1578.31 \pm 12.56$ & $1574.18 \pm 12.56$ & $1550.27 \pm 20.66$ \\
\hline $2 \times$ & $1535.94 \pm 42.12$ & $1579.05 \pm 28.97$ & $1527.72 \pm 30.71$ & $1578.29 \pm 12.68$ & $1520.76 \pm 30.68$ & $1558.50 \pm 20.83$ \\
\hline $4 \times$ & $1432.01 \pm 42.30$ & $1579.05+32.50$ & $1424.65 \pm 46.53$ & $1578.24 \pm 12.65$ & $1425.06 \pm 45.93$ & $1558.08 \pm 20.74$ \\
\hline $6 \times$ & $1381.02 \pm 43.17$ & $1579.04 \pm 36.76$ & $1377.20 \pm 48.83$ & $1578.19 \pm 12.62$ & $1380.53 \pm 47.95$ & $1549.50 \pm 25.53$ \\
\hline $8 \times$ & $1359.84 \pm 45.11$ & $1579.03 \pm 38.00$ & $1357.06 \pm 48.51$ & $1578.16 \pm 12.62$ & $1361.37 \pm 47.96$ & $1554.48 \pm 21.35$ \\
\hline $10 \times$ & $1346.82 \pm 45.87$ & $1579.04 \pm 40.47$ & $1344.87 \pm 46.94$ & $1578.21 \pm 12.65$ & $1350.34 \pm 46.86$ & $1554.12 \pm 21.53$ \\
\hline mean & 1439.10 & 1579.04 & 1434.97 & 1578.23 & 1435.37 & 1554.16 \\
\hline outlier & \multicolumn{6}{|c|}{ Expected Long-run Average Rewards (ElrAR) on the chain walk dataset } \\
\hline strength & LinUCB & Ro-LinUCB & $\mathrm{ACCB}$ & Ro-ACCB & SACCB & Ro-SACCB \\
\hline $0 \times$ & $47.061 \pm 6.714$ & $46.904 \pm 6.932$ & $52.191 \pm 4.545$ & $\mathbf{5 2 . 2 0 8} \pm \mathbf{4 . 5 5 4}$ & $52.215 \pm 4.669$ & $52.222 \pm 4.663$ \\
\hline $2 \times$ & $38.804 \pm 6.524$ & 47.217 \pm 7.071 & $39.407 \pm 5.395$ & $\mathbf{4 5 . 8 7 4} \pm \mathbf{4 . 9 7 4}$ & $38.756 \pm 5.468$ & $45.300 \pm 5.100$ \\
\hline $4 \times$ & $34.377 \pm 6.805$ & $49.398 \pm 6.751$ & $35.395 \pm 5.250$ & $\mathbf{4 9 . 2 2 5} \pm \mathbf{4 . 5 9 4}$ & $34.225 \pm 5.244$ & $48.216 \pm 4.950$ \\
\hline $6 \times$ & $31.458 \pm 6.589$ & $47.065 \pm 6.766$ & $34.909 \pm 5.072$ & $50.417 \pm 4.348$ & $34.012 \pm 5.128$ & $48.293 \pm 4.837$ \\
\hline $8 \times$ & $27.975 \pm 6.315$ & $42.550 \pm 7.030$ & $36.035 \pm 4.865$ & $49.499 \pm 4.760$ & $35.795 \pm 4.905$ & $\mathbf{4 7 . 4 5 5} \pm \mathbf{5 . 1 6 3}$ \\
\hline $10 \times$ & $25.085 \pm 5.831$ & $38.412 \pm 7.075$ & $37.431 \pm 4.684$ & $48.492 \pm 4.769$ & $37.171 \pm 4.698$ & $46.287 \pm 5.162$ \\
\hline mean & 34.127 & 45.257 & 39.228 & 49.286 & 38.696 & 47.962 \\
\hline
\end{tabular}

the long-run Heartsteps study when we use the estimated policy $\pi_{\widehat{\theta}_{n}}$ to send interventions to users. There are two steps to obtain the ElrAR: (a) get the average reward $\eta^{\pi_{\hat{\theta}_{n}}}$ for the $n^{\text {th }}$ user by averaging the rewards over the last 4,000 decision points in a trajectory of 5,000 tuples under the policy $\pi_{\hat{\theta}_{n}} ;(\mathrm{b})$ the $\operatorname{ElrAR} \mathbb{E}\left[\eta^{\pi} \hat{\theta}\right]$ is achieved

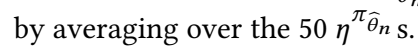

\subsection{Comparisons in the Four Experiment Settings}

We carry out the following experiments to verify four aspects of the contextual bandit methods:
(S1) To verify the significance of the proposed method, we compare nine contextual bandit methods on two datases: (1) HeartSteps and (2) chain walk. The experiment results are summarized in Table 1 , where there are three sub-tables; each sub-table displays three methods in a type: (a) the state-of-the-art contextual bandit, like ACCB; (b) "OutlierFilter + ACCB" means that we first use the state-of-the-art outlier filter [23, 28] to get rid of the outliers, then employ the state-of-the-art contextual bandit method ACCB for the SDM task; (c) is the proposed robust contextual bandit method (Ro-ACCB). As we shall see, "OutlierFilter" is helpful to improve the performance of the state-of-the-art contextual bandit methods. However, our three methods (i.e., Ro-LinUCB, Ro-ACCB and Ro-SACCB) always obtain the best results compared with all the 


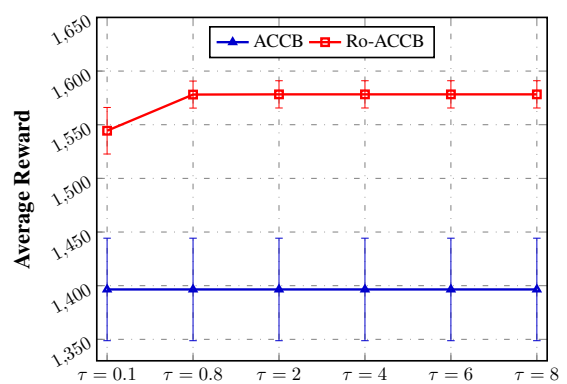

(a) Average reward vs. $\tau$ on HeartSteps

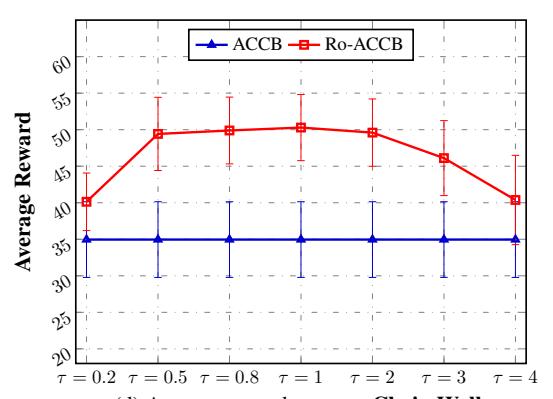

(d) Average reward vs. $\tau$ on Chain Walk

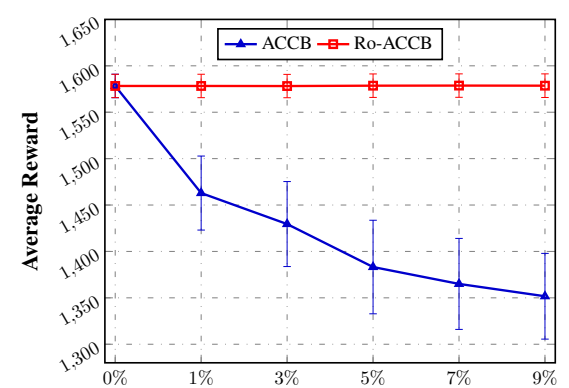

(b) Average reward vs. Outlier Ratio $\psi$ on HeartSteps

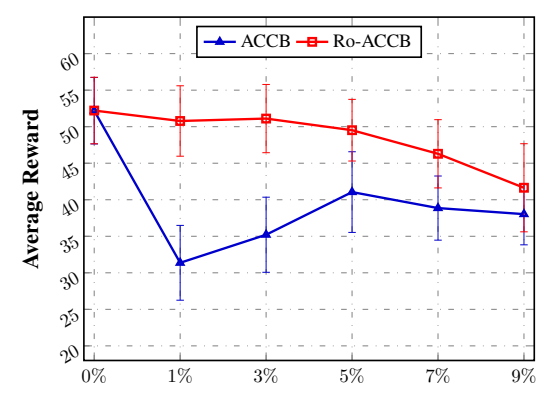

(e) Average reward vs. Outlier Ratio $\psi$ on Chain Walk

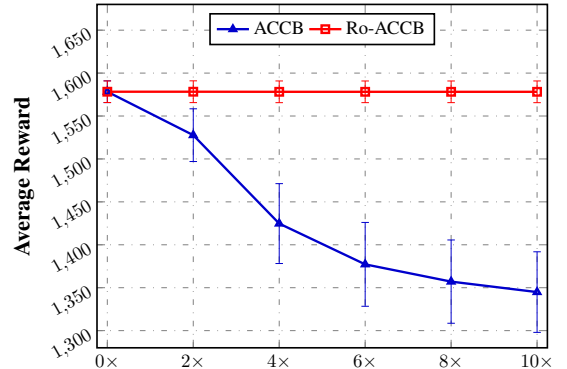

(c) Average reward vs. Outlier Strength $\nu$ on HeartSteps

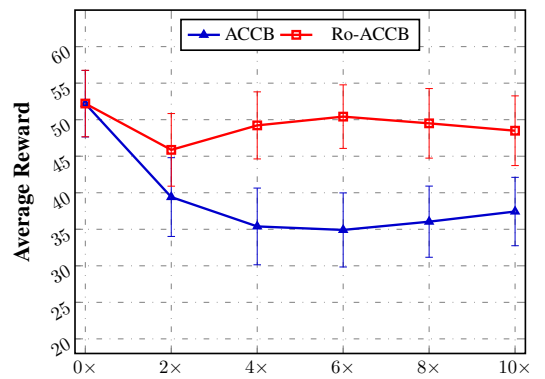

(f) Average reward vs. Outlier Strength $\nu$ on Chain Walk

Figure 1: The ElrAR of two contextual bandit methods $v$ s. $\epsilon$ (or $\tau$ in Eq. (5)), outlier ratio $\psi$ and outlier strength $v$ respectively on the two datasets. The top row of sub-figures illustrate the results on the HeartSteps, and the bottom row shows the results on the chain walk. The three columns of sub-figures show the result in the experiment setting (S4), (S2) and (S3) respectively.

other state-of-the-art methods in their type on both datasets. Compared with the best state-of-the-art method, our three methods improve 131.4, 136.2 and 139.9 steps respectively on the HeartSteps. Although there are lots of general outlier detection or outlier filter methods that can be helpful to relieve the bad influence of outliers, it is still meaningful to specifically propose a robust contextual bandit algorithm.

(S2) In this part, the ratio of tuples $\psi$ that contains outliers rises from $0 \%$ to $9 \%$. The experiment results are summarized in Table 2 and Figs. 1b, 1e. In Table 2, there are two sub-tables, displaying the ElrARs on the HeartSteps in the top, and the ElrARs on the chain walk in the bottom. As we can see, when $\mu=0 \%$, there is no outlier in the trajectory. In such case, our results are (almost) identical to that of LinUCB, ACCB and SACCB on both datasets. When $\psi$ rises, our results keep stable, while the ElrARs, of LinUCB, ACCB and SACCB, decrease dramatically. Compared with ACCB, Ro-ACCB on average achieves an improvement of $10.5 \%$ on the HeartSteps dataset and $23.2 \%$ on the chain walk dataset. Such results demonstrate that our method is able to deal with the badly noised dataset that consists of a large percentage (up to 9\%) of outliers.

(S3) In this part, the strength of outliers $v$ ranges from 0 to 10 times of the average value in the trajectory. The experiment results are summarized in Table 3, and Figs. 1c, 1f. We have three observations based on the experiment results: (1) when $v=0$, there is no outlier in the trajectory. Out methods achieve similar results with that of LinUCB, ACCB and SACCB; (2) when $v$ rises in the domain, our method keeps stable on the HeartSteps and decreases slightly on the chain walk. However, the results of LinUCB, ACCB and SACCB decrease obviously when $v$ rises. Such phenomena verify that our method is able to deal with the dataset both with or without outliers. Besides, we may use our method on the dataset with various strengths of outliers.

(S4) In this part, the value of $\tau$ ranges from 0.1 to 8 on the HeartSteps dataset and from 0.2 to 4 on the chain walk dataset. The experiment results are displayed in Figs. 1a and 1d. As we shall see, the proposed method obtains clear advantage over the state-of-theart method, i.e., ACCB [17], in a wide range of $\tau$ settings. In average, our method improves the ElrAR by $12.6 \%$ on the HeartSteps and $33.1 \%$ on the chain walk, compared with the ACCB. Such results verify that the proposed method to set $\tau$ is very promising. It is able to adapt to the data property and select the effective, neither too few nor too many, tuples in the trajectory for the actor-critic updating. Note that ACCB does not have the parameter $\tau$. Thus the result of ACCB remains unchanged as $\tau$ rises.

\section{CONCLUSION AND DISCUSSION}

To deal with the outlier in the trajectory, we propose a robust actorcritic contextual bandit for the mHealth intervention. The capped- $\ell_{2}$ norm is employed to boost the robustness for the critic updating. With the learned weights in the critic updating, we propose a new objective for the actor updating, enhancing its robustness. Besides, we propose a solid method to set an important thresholding parameter in the capped- $\ell_{2}$ norm. With it, we can achieve the conflicting goal of boosting the robustness of our algorithm on the dataset with outliers, and achieving almost identical results compared with the state-of-the-art method on the datasets without outliers. Besides, we provide theoretical guarantee for our algorithm. It shows that 
our algorithm could find sufficiently decreasing point after each iteration and finally converges after a finite number of iterations. Extensive experiment results show that in a variety of parameter settings our method achieves significant improvements.

\section{Appendix 1: the proof of Proposition 1}

Proof. According to the analyses in Eqs (6) and (10), we simplify (4) into the following objective

$$
\min _{\mathbf{w}} \sum_{i=1}^{M} u_{i}\left\|r_{i}-\mathbf{x}_{i}^{\top} \mathbf{w}\right\|_{2}^{2}+\zeta_{c}\|\mathbf{w}\|_{2}^{2} .
$$

Let $\mathbf{U} \in \mathbb{R}^{\boldsymbol{T}^{\times} \mathrm{T}}$ be a non-negative diagonal matrix, with the $i^{\text {th }}$ diagonal element as

$$
U_{i i}=1_{\left\{\left\|r_{i}-\mathbf{x}_{i}^{T} \mathbf{w}\right\|_{2}^{2}<\epsilon\right\}} .
$$

Taking the partial derivative of (15) with respect to $\mathbf{w}$ and setting the derivative to zero give us the updating rule

$$
\widehat{\mathbf{w}}^{(t)}=\left(\mathrm{XU}^{(t-1)} \mathbf{X}^{\boldsymbol{\top}}+\zeta \mathbf{I}\right)^{-1} \mathbf{X U}^{(t-1)} \mathbf{r},
$$

where

$$
\mathbf{U}^{(t)}=\operatorname{diag}\left(\mathbf{u}^{(t)}\right) \in \mathbb{R}^{M \times M}
$$

is non-negative diagonal matrix, whose $i^{\text {th }}$ element is

$$
u_{i}^{(t)}=1_{\left\{\left\|r_{i}-\mathbf{x}_{i}^{\top} \widehat{\mathbf{w}}^{(t)}\right\|_{2}^{2}<\epsilon\right\}} .
$$

\section{Appendix 2: the proof of Lemma 3.1}

Proof. For $t \geq 1$, when fix $\mathbf{u}^{(t-1)}$, we find that

$$
\begin{aligned}
F\left(\mathbf{w}, \mathbf{u}^{(t-1)}\right)= & \sum_{i}^{M} u_{i}^{(t-1)}\left\|r_{i}-\mathbf{x}_{i}^{T} \mathbf{w}\right\|_{2}^{2}+ \\
& \sum_{i}^{M}\left(1-u_{i}^{(t-1)}\right) \epsilon+\zeta_{c}\|\mathbf{w}\|_{2}^{2}
\end{aligned}
$$

is a quadratic function in $\mathbf{w}$, which is also strongly convex in w. So, the updating rule in Proposition 1, based on solving the gradient of $F\left(\mathbf{w}, \mathbf{u}^{(t-1)}\right)$ equals zero, minimizes $F\left(\mathbf{w}, \mathbf{u}^{(t-1)}\right)$ globally over $\mathbf{w}$. The strong convexity of $F\left(\mathbf{w}, \mathbf{u}^{(t-1)}\right)$ at $\mathbf{w}^{(t)}$ says that, for all $\mathbf{w} \in \mathbb{R}^{u}$,

$$
\begin{aligned}
F\left(\mathbf{w}, \mathbf{u}^{(t-1)}\right) & \geq F\left(\mathbf{w}^{(t)}, \mathbf{u}^{(t)}\right)+ \\
& \left\langle\nabla F\left(\mathbf{w}^{(t)}, \mathbf{u}^{(t-1)}\right), \mathbf{w}-\mathbf{w}^{(t)}\right\rangle+\zeta_{c}\left\|\mathbf{w}-\mathbf{w}^{(t)}\right\|_{2}^{2}
\end{aligned}
$$

Now, let $\mathbf{w}=\mathbf{w}^{(t-1)}$, and notice that

$$
\nabla F\left(\mathbf{w}^{(t)}, \mathbf{u}^{(t-1)}\right)=0
$$

in the above, we have

$$
F\left(\mathbf{w}^{(t-1)}, \mathbf{u}^{(t-1)}\right) \geq F\left(\mathbf{w}^{(t)}, \mathbf{u}^{(t-1)}\right)+\zeta_{c}\left\|\mathbf{w}^{(t)}-\mathbf{w}^{(t-1)}\right\|_{2}^{2} .
$$

When fixing $\mathbf{w}^{(t)}$, updating $\mathbf{u}^{(t)}$ gives

$$
\begin{aligned}
& F\left(\mathbf{w}^{(t)}, \mathbf{u}^{(t)}\right)-F\left(\mathbf{w}^{(t)}, \mathbf{u}^{(t-1)}\right) \\
= & \sum_{i}^{M}\left(u_{i}^{(t)}-u_{i}^{(t-1)}\right)\left\|r_{i}-\mathbf{x}_{i}^{T} \mathbf{w}^{(t)}\right\|_{2}^{2} \\
& -\sum_{i}^{M}\left(u_{i}^{(t)}-u_{i}^{(t-1)}\right) \epsilon \\
= & \sum_{i}^{M}\left(u_{i}^{(t)}-u_{i}^{(t-1)}\right)\left(\left\|r_{i}-\mathbf{x}_{i}^{T} \mathbf{w}^{(t)}\right\|_{2}^{2}-\epsilon\right)
\end{aligned}
$$

Recall that we define

$$
u_{i}^{(t)}=1_{\left\{\left\|r_{i}-\mathbf{x}_{i}^{\top} \widehat{\mathbf{w}}^{(t)}\right\|_{2}^{2}<\epsilon\right\}} .
$$

Apply this definition to (5) we have:

$$
\begin{aligned}
& F\left(\mathbf{w}^{(t)}, \mathbf{u}^{(t)}\right)-F\left(\mathbf{w}^{(t)}, \mathbf{u}^{(t-1)}\right) \\
= & -\sum_{i}^{M}\left|\left\|r_{i}-\mathbf{x}_{i}^{\top} \mathbf{w}\right\|_{2}^{2}-\epsilon\right| 1_{\left\{u_{i}^{(t)} \neq u_{i}^{(t-1)}\right\}} \leq 0 .
\end{aligned}
$$

From the above analysis, we see that updating both $\mathbf{w}$ and $\mathbf{u}$ according to our rule in proposition 1 will lead to decrease of value in objective function, for which we are trying to minimize. Thus $\forall t \geq 1$, we conclude that the proposed updating rule will sufficiently decrease the objective function value along the iterations, "sufficiency" refers to the positive gap of function value reduction when updating w. To summarize, we have sufficient decrease property:

$$
\begin{aligned}
& F\left(\mathbf{w}^{(t-1)}, \mathbf{u}^{(t-1)}\right) \\
\geq & F\left(\mathbf{w}^{(t)}, \mathbf{u}^{(t)}\right)+\zeta_{c}\left\|\mathbf{w}^{(t)}-\mathbf{w}^{(t-1)}\right\|_{2}^{2} .
\end{aligned}
$$

\section{Appendix 3: the proof of Lemma 3.2}

Proof. We sum up the function descent inequality (16) for $t=$ $1,2, \cdots, T(O(\mathbf{w}) \equiv F(\mathbf{w}, \mathbf{u}))$ :

$$
\begin{aligned}
& \sum_{t=1}^{T}\left\|\mathbf{w}^{(t)}-\mathbf{w}^{(t-1)}\right\|_{2}^{2} \\
\leq & \frac{1}{\zeta_{c}} \sum_{i=1}^{T}\left[O\left(\mathbf{w}^{(t-1)}\right)-O\left(\mathbf{w}^{(t)}\right)\right] \\
= & \frac{1}{\zeta_{c}}\left[O\left(\mathbf{w}^{(0)}\right)-O\left(\mathbf{w}^{(T)}\right)\right] .
\end{aligned}
$$

From (16), the sequence $\left\{O\left(\mathbf{w}^{(t)}\right), t \geq 0\right\}$ is non increasing with $O(\mathbf{w}) \geq 0, \forall \mathbf{w} \in \mathcal{W}$. Taking the limit of $T \rightarrow \infty$ on both sides of (19), we get

$$
\sum_{t=1}^{\infty}\left\|\mathbf{w}^{(t)}-\mathbf{w}^{(t-1)}\right\|_{2}^{2} \leq \infty
$$

and thus

$$
\lim _{t \rightarrow \infty}\left\|\mathbf{w}^{(t)}-\mathbf{w}^{(t-1)}\right\|_{2}^{2}=0 .
$$


The above Lemma 3.2 actually has shown that our updating rule results in a sequence $\mathbf{w}^{(t)}$ which either converges or has its accumulation points in a continuum. We will show it converges in our case in the following.

With Lemma 3.1 and Lemma 3.2, one can actually show that, given a fixed outlier thresholding $\epsilon>0$, the algorithm converges after finite number of iterations.

\section{Appendix 4: the proof of Theorem 1}

Proof. We first show that the sequence $\left\{O\left(\mathbf{w}^{(t)}\right), t \in \mathbb{N}\right\}$ is bounded. Although the least squared loss function

$$
h_{i}(\mathbf{w})=\left\|r_{i}-\mathbf{x}_{i}^{\top} \mathbf{w}\right\|_{2}^{2}
$$

maps an unbounded domain to an unbounded range, in our problem, it is capped with a finite $\epsilon>0$ term. That is, if

$$
\min _{i=1, \cdots, M}\left\{\left\|r_{i}-\mathbf{x}_{i}^{\top} \mathbf{w}^{(\tau)}\right\|_{2}^{2}\right\}>\epsilon,
$$

at a certain update step $\tau$, then the objective function will be reduced to include only the regularization term $\zeta_{c}\|\mathbf{w}\|_{2}^{2}$ to minimize in the next step. This will result in the critic update (the updating rule in Proposition 1) stop with $\mathbf{w}^{(t)}=0, \forall t \geq \tau$. So the sequence

$$
\left\{O\left(\mathbf{w}^{(t)}\right), t \in \mathbb{N}\right\}
$$

must be bounded such that $\left\|\mathbf{w}^{(t)}\right\|_{2}^{2} \leq M$, for some constant $M>0$.

Now $\forall t \geq 1$, we have

$$
\begin{aligned}
&\left\|r_{i}-\mathbf{x}_{i}^{\top} \mathbf{w}^{(t)}\right\|_{2}^{2}-\left\|r_{i}-\mathbf{x}_{i}^{\top} \mathbf{w}^{(t-1)}\right\|_{2}^{2} \mid \\
& \leq B\left\|\mathbf{w}^{(t)}-\mathbf{w}^{(t-1)}\right\|_{2}^{2}
\end{aligned}
$$

for some constant $B>0$. Then via Lemma 3.2 which says that

$$
\lim _{t \rightarrow \infty}\left\|\mathbf{w}^{(t)}-\mathbf{w}^{(t-1)}\right\|_{2}^{2}=0 \text {. }
$$

, thus for a given fixed outlier threshold parameter $\epsilon>0$, we deduce that there exists $T \in \mathbb{N}$, such that when $t \geq T$, we have

$$
\left(\left\|r_{i}-\mathbf{x}_{i}^{\top} \mathbf{w}^{(t)}\right\|_{2}^{2}-\epsilon\right) \cdot\left(\left\|r_{i}-\mathbf{x}_{i}^{\top} \mathbf{w}^{(t-1)}\right\|_{2}^{2}-\epsilon\right) \geq 0
$$

$\forall i=1, \cdots, T$. That is $\forall i=1, \cdots, T, u_{i}$ will remain unchanged for all $t \geq T$ and the problem is reduced to a least square problem (or ridge regression problem specifically), in which only $\mathbf{w}^{(T)}$ will be finally updated by an one step closed solution like a ridge regression solution. Thus after $T+1<\infty$ steps, the updating rule in Proposition 1 , which stems from solving a gradient equals zero equation, will converge to at least to a local minimum of the problem (4), with a closed form solution:

$$
\widehat{\mathbf{w}}^{(t)}=\left(\mathrm{XU}^{(t-1)} \mathrm{X}^{\boldsymbol{\top}}+\zeta \mathrm{I}\right)^{-1} \mathrm{XU}^{(t-1)} \mathbf{r} .
$$

This conclusion is in general the best convergence result one can hope for a non-convex minimization problem in general.

\section{REFERENCES}

[1] King Abby, Hekler Eric, Grieco Lauren, Winter Sandra, Sheats Jylana, Buman Matthew, .., and Cirimele Jesse. 2013. Harnessing different motivational frames via mobile phones to promote daily physical activity and reduce sedentary behavior in aging adults. Plos ONE 8, 4 (2013).
[2] D. Ben-Zeev, K. E. Davis, S. Kaiser, I. Krzsos, and R. E. Drake. 2013. Mobile technologies among people with serious mental illness: opportunities for future services. Administration and Policy in Mental Health and Mental Health Services Research 40, 4 (2013), 34-343.

[3] Ku-Chun Chou, Hsuan-Tien Lin, Chao-Kai Chiang, and Chi-Jen Lu. 2014. Pseudoreward Algorithms for Contextual Bandits with Linear Payoff Functions. In $7 M L R$ : Workshop and Conference Proceedings. 1-19.

[4] Robert Dawson. 2011. How significant is a boxplot outlier. Fournal of Statistics Education 19, 2 (2011), 1-12.

[5] Walter Dempsey, Peng Liao, Pedja Klasnja, Inbal Nahum-Shani, and Susan A. Murphy. 2016. Randomised trials for the Fitbit generation. Significance 12, 6 (Dec 2016), $20-23$

[6] Lee Dicker. 2014. Sparsity and the truncated 1^ 2-norm. In Artificial Intelligence and Statistics. 159-166.

[7] Miroslav Dudík, John Langford, and Lihong Li. 2011. Doubly Robust Policy Evaluation and Learning. In ICML. 1097-1104.

[8] Joseph Firth, John Torous, and Alison Yung. 2016. Ecological momentary assessment and beyond: the rising interest in e-mental health research. Fournal of psychiatric research 80 (2016), 3-4.

[9] Hongchang Gao, Feiping Nie, Weidong Cai, and Heng Huang. 2015. Robust Capped Norm Nonnegative Matrix Factorization: Capped Norm NMF. In ACM International Conference on Information and Knowledge. 871-880.

[10] Ivo Grondman, Lucian Busoniu, Gabriel A. D. Lopes, and Robert Babuska. 2012. A Survey of Actor-Critic Reinforcement Learning: Standard and Natural Policy Gradients. IEEE Trans. Systems, Man, and Cybernetics 42, 6 (2012), 1291-1307.

[11] D.H. Gustafson, F.M. McTavish, M.Y. Chih, A.K. Atwood, ..., and D. Shah. 2014 A smartphone application to support recovery from alcoholism: a randomized clinical trial. FAMA Psychiatry 71, 5 (2014), 566-572.

[12] Chun-Yen Ho and Hsuan-Tien Lin. 2015. Contract Bridge Bidding by Learning.. In AAAI Workshop: Computer Poker and Imperfect Information.

[13] Wenhao Jiang, Feiping Nie, and Heng Huang. 2015. Robust Dictionary Learning with Capped 11-Norm.. In IFCAI. 3590-3596.

[14] Predrag Klasnja, Eric B Hekler, Saul Shiffman, Audrey Boruvka, Daniel Almirall, Ambuj Tewari, and Susan A Murphy. 2015. Microrandomized trials: An experimental design for developing just-in-time adaptive interventions. Health Psychology 34, S (2015), 1220.

[15] Michail G Lagoudakis and Ronald Parr. 2003. Least-squares policy iteration. Journal of machine learning research 4, Dec (2003), 1107-1149.

[16] Huitian Lei. 2016. An Online Actor Critic Algorithm and a Statistical Decision Procedure for Personalizing Intervention. Ph.D. Dissertation. University of Michigan.

[17] Huitian Lei, A. Tewari, and Susan Murphy. 2014. An Actor-Critic Contextual Bandit Algorithm for Personalized Interventions using Mobile Devices. In NIPS 2014 Workshop: Personalization: Methods and Applications. 1 - 9.

[18] Lihong Li, Wei Chu, John Langford, and Robert E. Schapire. 2010. A contextualbandit approach to personalized news article recommendation. In International Conference on World Wide Web (WWW). 661-670.

[19] Ruoyu Li and Junzhou Huang. 2015. Fast regions-of-interest detection in whole slide histopathology images. In International Workshop on Patch-based Techniques in Medical Imaging. Springer, 120-127.

[20] Ruoyu Li, Sheng Wang, Feiyun Zhu, and Junzhou Huang. 2018. Adaptive Graph Convolutional Neural Networks. arXiv:1801.03226 (2018).

[21] Xiujun Li, Lihong Li, Jianfeng Gao, Xiaodong He, Jianshu Chen, Li Deng, and Ji He. 2015. Recurrent reinforcement learning: a hybrid approach. arXiv:1509.03044 (2015).

[22] Peng Liao, A. Tewari, and Susan Murphy. 2015. Constructing Just-in-Time Adaptive Interventions. Phd Section Proposal (2015), 1-49.

[23] Hancong Liu, Sirish Shah, and Wei Jiang. 2004. On-line outlier detection and data cleaning. Computers \& chemical engineering 28, 9 (2004), 1635-1647.

[24] Susan A. Murphy, Yanzhen Deng, Eric B. Laber, Hamid Reza Maei, Richard S. Sutton, and Katie Witkiewitz. 2016. A Batch, Off-Policy, Actor-Critic Algorithm for Optimizing the Average Reward. CoRR abs/1607.05047 (2016).

[25] Feiping Nie, Heng Huang, Xiao Cai, and Chris H. Ding. 2010. Efficient and Robust Feature Selection via Joint $\ell_{2,1}$-Norms Minimization. In NIPS. 1813-1821.

[26] Jorge Nocedal and Stephen J. Wright. 2006. Numerical Optimization (2nd ed.). Springer, New York.

[27] Qian Sun, Shuo Xiang, and Jieping Ye. 2013. Robust principal component analysis via capped norms. In ACM SIGKDD. 311-319.

[28] J Suomela. 2014. Median Filtering is Equivalent to Sorting. arXiv:1406.1717 (2014).

[29] Richard S. Sutton and Andrew G. Barto. 2012. Reinforcement Learning: An Introduction (2nd ed.). MIT Press, Cambridge, MA, USA.

[30] Ambuj Tewari and Susan A. Murphy. 2017. From Ads to Interventions: Contextual Bandits in Mobile Health. In Mobile Health: Sensors, Analytic Methods, and Applications, Jim Rehg, Susan A. Murphy, and Santosh Kumar (Eds.). Springer.

[31] Ying Wang, Chunhong Pan, Shiming Xiang, and Feiyen Zhu. 2015. Robust hyperspectral unmixing with correntropy-based metric. IEEE Transactions on Image Processing 24, 11 (2015), 4027-4040. 
[32] David F Williamson, Robert A Parker, and Juliette S Kendrick. 1989. The box plot: a simple visual method to interpret data. Annals of internal medicine 110, 11 (1989), 916-921.

[33] K. Witkiewitz, S. Desai, S. Bowen, B. Leigh, M. Kirouac, and M. Larimer. 2014 Development and evaluation of a mobile intervention for heavy drinking and smoking among college studen. Psychology of Addictive Behaviors 28, 3 (2014), 639-650.

[34] Huan Xu. 2009. Robust decision making and its applications in machine learning. McGill University.

[35] Zheng Xu and Junzhou Huang. 2016. Detecting 10,000 cells in one second. In International Conference on Medical Image Computing and Computer-Assisted Intervention. Springer, 676-684.

[36] Zheng Xu, Sheng Wang, Feiyun Zhu, and Junzhou Huang. 2017. Seq2seq fin gerprint: An unsupervised deep molecular embedding for drug discovery. In Proceedings of the 8th ACM International Conference on Bioinformatics, Computational Biology, and Health Informatics. ACM, 285-294.

[37] Min Yang, Linli Xu, Martha White, Dale Schuurmans, and Yao-liang Yu. 2010 Relaxed clipping: A global training method for robust regression and classification. In Advances in neural information processing systems. 2532-2540.
[38] Baichuan Zhang and Mohammad Al Hasan. 2017. Name Disambiguation in Anonymized Graphs using Network Embedding. In Proceedings of the 26th ACM International on Conference on Information and Knowledge Management.

[39] Baqun Zhang, Anastasios A Tsiatis, Eric B Laber, and Marie Davidian. 2012. A robust method for estimating optimal treatment regimes. Biometrics 68, 4 (2012), 1010-1018.

[40] Feiyun Zhu, Bin Fan, Xinliang Zhu, Ying Wang, Shiming Xiang, and Chunhong Pan. 2015. 10,000+ Times Accelerated Robust Subset Selection (ARSS). In Proc. Assoc. Adv. Artif. Intell. (AAAI). 3217-3224.

[41] Feiyun Zhu, Jun Guo, Zheng Xu, Peng Liao, and Junzhou Huang. 2018. Groupdriven Reinforcement Learning for Personalized mHealth Intervention. In International Conference on Medical Image Computing and Computer Assisted Intervention

[42] Feiyun Zhu and Peng Liao. 2017. Effective warm start for the online actor-critic reinforcement learning based mhealth intervention. In The Multidisciplinary Conference on. Reinforcement Learning and Decision Making.

[43] Feiyun Zhu, Ying Wang, Bin Fan, Gaofeng Meng, and Chunhong Pan. 2014. Effective Spectral Unmixing via Robust Representation and Learning-based Sparsity. CoRR abs/1409.0685 (2014). http://arxiv.org/abs/1409.0685 